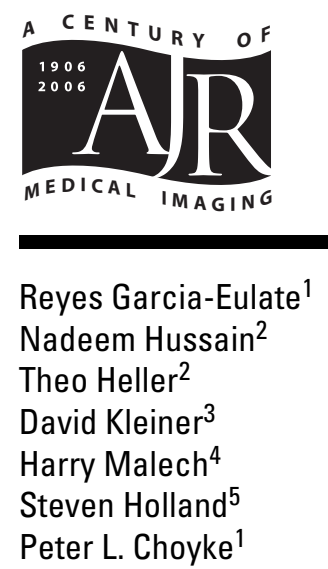

Keywords: CT, genetics, infectious diseases, liver disease, MRI

DOI:10.2214/AJR.05.1386

Received August 9, 2005; accepted after revision February 28, 2006.

This research was supported in part by the Intramural Research Program of the National Institutes of Health (NIH), National Cancer Institute, and Center for Cancer Research.

${ }^{1}$ Molecular Imaging Program, National Cancer Institute, Bldg. 10, Rm. B3B69, 9000 Rockville Pike, Bethesda, MD 20892. Address correspondence to P. L. Choyke (pchoyke@nih.gov).

${ }^{2}$ Liver Diseases Branch, National Institute of Diabetes and Digestive and Kidney Diseases, Bethesda, MD.

${ }^{3}$ Laboratory of Pathology, National Cancer Institute, Bethesda, MD.

${ }^{4}$ Laboratory of Host Defenses, National Institute of Allergy and Infectious Diseases, Bethesda, MD.

${ }^{5}$ Laboratory of Clinical Infectious Diseases, Bethesda, MD.

\section{CME}

This article is available for is available for 1 CME credit. See CME data for this article at www.ajronline.org or visit www.arrs.org for more information.

AJR 2006; 187:482-490

0361-803X/06/1872-482

(C) American Roentgen Ray Society

\title{
$C T$ and MRI of Hepatic Abscess in Patients with Chronic Granulomatous Disease
}

OBJECTIVE. We describe the spectrum of radiologic appearances of hepatic abscesses in patients with chronic granulomatous disease (CGD), a hereditary immunodeficiency presenting in childhood that occurs at a rate of 1 in 200,000-250,000 live births and predisposes patients to infection with catalase-positive organisms.

CONCLUSION. Hepatic abscesses in patients with CGD show an atypical radiologic appearance compared with sporadic hepatic abscesses, and they are characterized by homogeneous enhancement and multiseptal enhancement. In the appropriate clinical setting, the appearance of an enhancing mass should suggest the possibility of a CGD-related hepatic abscess.

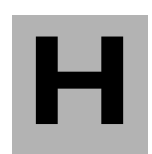
epatic abscesses are usually caused by a bacterial infection associated with ascending cholangitis or trauma, among other causes, and generally they have a well-defined appearance on CT and MRI. Abscesses typically display a thin enhancing rim with liquefied nonenhancing internal contents [1-3]. Histology reveals active inflammatory cells in the periphery with necrosis and pus in the center $[1,4]$. Hepatic abscesses typically show progressive liquefaction at their center over the course of 2 weeks [5]. Because of their liquid contents, hepatic abscesses typically respond to a combination of parenteral antibiotics, percutaneous or open drainage, or both.

Chronic granulomatous disease (CGD) is the name used to describe a group of rare immune deficiencies caused by mutations in one of four genes that encode subunits of phagocytic nicotinamide adenine dinucleotide phosphate (NADPH) oxidase [6-8]. This disease occurs once in 200,000-250,000 live births. Although survival into the fourth and fifth decades is now more common because of aggressive antibiotic therapy, life expectancy is typically reduced in most patients. Patients with CGD are susceptible to catalase-positive microorganisms such as Staphylococcus aureus and fungi that metabolize their own hydrogen peroxide [7-9]. Recurrent infections, especially in the lungs, with certain bacteria (Staphylococcus aureus, Serratia marcescens, Burkholderia cepacia, and Nocardia species) and fungi (Aspergillus species and others) are common. In addition, these patients develop recurrent inflammatory lesions, characteristically involving hollow viscera. CGD is diagnosed by showing a defective oxidative burst in lymphocytes as shown by nitroblue tetrazolium (NBT) reduction or dihydror hodamine oxidation.

Instead of forming normal liquefied abscesses, these CGD-associated abscesses show granulomatous inflammation with a dense surrounding granulation tissue $[8,10]$. Granulomatous abscesses typically contain an intense leukocytic infiltrate centrally and granulation or reparative tissue peripherally. Liver abscesses occur in approximately 50\% of the patients and are typically asymptomatic or accompanied only by fever. Pain is less frequent, and liver enzyme elevations are inconsistent and unhelpful. CGD-associated abscesses thus are difficult to diagnose and do not typically liquefy, making percutaneous drainage difficult or impossible [11].

The spectrum of CT and MRI appearances of hepatic abscess in CGD has not received much attention in the literature. We report the CT and MRI appearance of hepatic abscesses in 24 patients with CGD obtained over a 23-year period. We review the radiologic appearance of hepatic abscesses in CGD on CT and MRI.

\section{Materials and Methods}

We conducted a retrospective review of the imaging studies of 47 patients with CGD between 1981 and 2004, selected from a cohort of 194 patients who were entered in a protocol approved by 


\section{Imaging of Hepatic Abscess in Chronic Granulomatous Disease}

the institutional review board and from whom informed consent was obtained. Selection criteria included history of liver biopsy, known history of liver abscess, or progressive thrombocytopenia that was likely related to splenomegaly. From this population, 24 patients were found to have clinical imaging and pathologic evidence of one or more hepatic abscesses. All cases of suspected hepatic abscess were confirmed with either percutaneous aspiration, surgery, or both, and they had positive bacteriologic or fungal cultures. However, if a patient had one positive aspiration, any remaining hepatic lesions were also assumed to be an abscess.

Among the 24 patients were 22 males (92\%) and two females $(8 \%)$. The predominance of males reflects the fact that most cases of CGD are X-linked. At the onset of the abscess, the age range was 3-31 years with a mean of 13.4 years.

A hepatic abscess episode was defined as a presentation with signs and symptoms of infection ultimately diagnosed as a hepatic abscess by histologic or microbiologic criteria. Because CGD patients are prone to recurrent infections, the number of episodes of infection and abscesses far exceeds the number of patients.

In most patients, CT was used as a diagnostic method and MRI was reserved for patients with allergies or renal insufficiency. Thus, 113 abscesses were imaged with CT and only 50 were imaged with MRI. Twelve patients had both MRI and CT during the same episode. In older patients who did not require sedation, MRI was used to confirm suspected findings on CT. Because of the duration of this review (1981-2004), several generations of imaging equipment were used. CT scans ranged from singledetector nonhelical to 16-MDCT. Contrast medium was used (120-140 mL iopamidol [Isovue 300, Bristol-Myers Squibb] after 1986) in all patients except if contraindicated by a history of allergy.

Similarly, the quality and type of MRI varied considerably over the duration of this study. MRI ranged from whole-body coil imaging at $0.5 \mathrm{~T}$ (Vista, Picker International) to torso coil imaging at $1.5 \mathrm{~T}$ (Signa, GE Healthcare). Early studies were performed at $0.5 \mathrm{~T}$ using STIR with the following parameters: TR/TE, 1,500/30; and inversion time (TI), 100 milliseconds. T2-weighted MRI was performed with 2,000/80, and T1weighted MRI was performed with 500/10. Later in the study, the following sequences were performed on 1.5-T scanners (Signa Horizon, GE Healthcare): a STIR sequence with a TR/TE range of 2,000/25-30 and a TI of 150 milliseconds; T2weighted fast spin-echo fat-suppressed sequence with a TR range/TE of 3,000-4,000/102 and echo train of 4-6; and T1-weighted gradient-echo sequence with a TR/TE of 150/4.2 (before and after
IV contrast agent administration using 0.1 $\mathrm{mmol} / \mathrm{kg}$ gadopentetate dimeglumine after 1988).

In summary, 34 of the $113 \mathrm{CT}$ scans were obtained before the helical CT era, 35 were obtained during the single-detector helical era, and the remaining 44 scans were obtained using a 4- to 16MDCT unit. For MRI, 15 examinations were performed on 0.5 - $\mathrm{T}$ scanners and 35 on 1.5 -T scanners. Note that the protocol for abscesses on MRI (T1and T2-weighted or STIR scans followed by a nondynamic enhanced fat-suppressed T1-weighted scan) is not particularly demanding and has been essentially unchanged for the last 10 years. Thus, a substantial fraction, 39\% for CT and 70\% for MRI, were obtained with modern equipment.The CT and MRI results were reviewed independently by two board-certified radiologists who were blinded to each other's interpretations, and consensus was reached regarding the imaging characteristics using a case report form.

\section{Results}

Twenty-four of 47 patients (51\%) showed hepatic abscesses. A total of 151 abscesses were found in 53 patient episodes, for a mean of 2.9 abscesses per episode. Followup ranged from 1 to 20 years, with a mean of 10.6 years. It consisted of a combination of clinical examination and imaging studies. The length of follow-up was confirmed in each patient by chart review.

\section{Overall Description}

Abscesses of the liver ranged in size from smaller than $1 \mathrm{~cm}$ to $14 \mathrm{~cm}$ in diameter (mean, $3.0 \mathrm{~cm}$ ). The number of episodes per patient ranged from 1 to 8 . The number of abscesses per patient ranged from 1 to more than 10 . Fourteen patients had only 1 episode, and 10 had more than 1 . In 20 episodes, only 1 abscess appeared; in 33 episodes, more than 1 abscess was diagnosed. Among recurrent cases of abscess, five patients had a solitary abscess and 19 had multiple abscesses (Fig. 1). Lesions were found in both the right and left lobes of the liver, without any specific distribution. Histologically, abscesses in the liver in CGD are irregular and surrounded by layered inflammation. Frequently, several such abscesses appear within any given hepatic lesion. The center of the abscess is filled with neutrophils and cell debris, and this core is surrounded by palisade epithelioid histiocytes and then by a layer of histiocytes mixed with other inflammatory cells (mainly lymphocytes). Around this is a wide zone of fibrous and inflamed granulation tissue. In the periphery of the abscess are numerous small vessels with activated endothelium within a fibrous stroma. The intensity of inflammation is less than that immediately adjacent to the abscess. Lymphocytes, eosinophils, and macrophages are usually present in this region (Fig. 2).

\section{Imaging Findings}

Among the 151 abscesses, 113 underwent CT and 50 underwent MRI (12 patients had both studies). The appearance of hepatic abscess varied widely, from homogeneously enhancing lesions to highly heterogeneous lesions with decreased attenuation on unenhanced CT scans. The lesions had sharply defined margins. The patterns of enhancement could be divided into three types

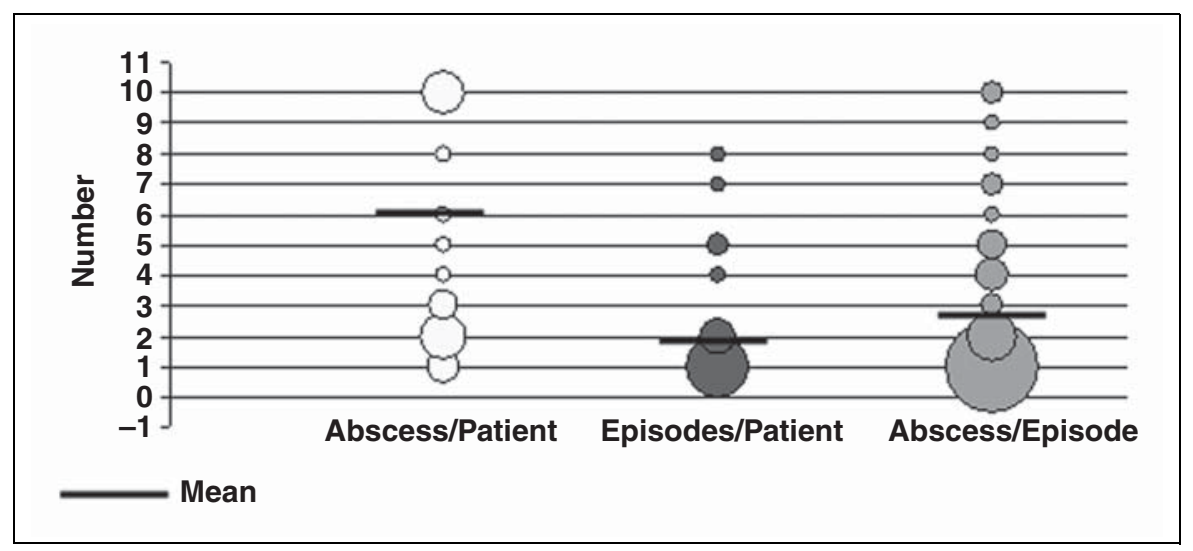

Fig. 1-Scatter chart showing number of abscesses per patient, number of episodes of abscess per patient, and number of abscesses per episode. $Y$-axis represents numerator of each ratio, and size of circle represents denominator. Number of abscesses per patient has bimodal distribution with most patients having either 1 or 2 or 10 or more. Most patients had only a few episodes and only a few abscesses ( 1 or 2 ) per episode, but some patients showed numerous episodes with multiple abscesses. 


\section{Garcia-Eulate et al.}
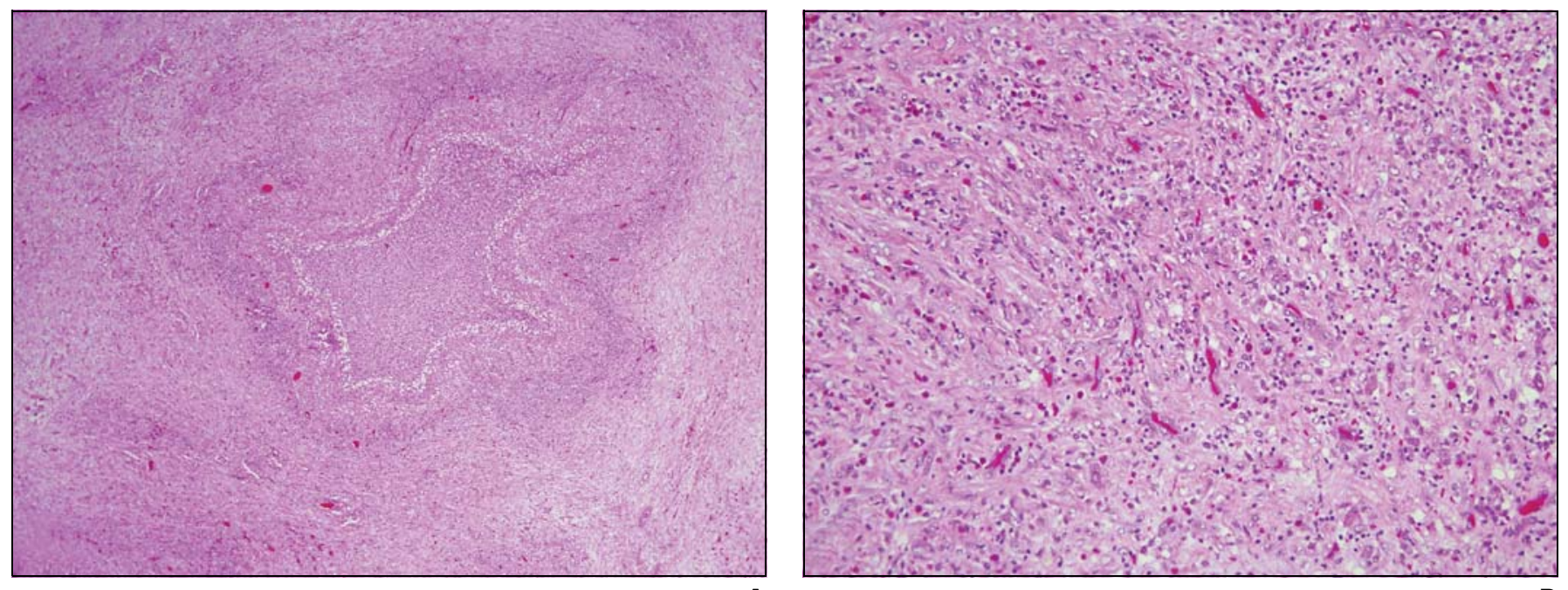

Fig. 2-16-year-old boy with chronic granulomatous disease (CGD) shows typical histologic appearance of hepatic abscess.

A, Unlike most abscesses, center of abscess in CGD is filled with neutrophils and cell debris, and this core is surrounded by palisade epithelioid histiocytes and then by layer of histiocytes mixed with other inflammatory cells (mainly lymphocytes). Around this is wide zone of fibrous and inflamed granulation tissue. Typical abscesses showed liquified necrosis centrally. ( $H$ and $E, \times 40)$

B, Higher magnification of vascular granulation tissue surrounding abscess shows numerous small vessels with activated endothelium within fibrous stroma. Lymphocytes, eosinophils, and macrophages are present in this region as well. $(H$ and $E, \times 200)$

(Fig. 3): type 1, complete central enhancement (i.e., entirely solid) $(n=41)$; type 2 , incomplete central enhancement subdivided into type $2 \mathrm{a}$, which showed a small central area with poor enhancement $(n=71)$, and type $2 b$, which showed peripheral enhancement and a multiseptal core enhancement, suggesting a multiloculated abscess $(n=22)$; and type 3, no central enhancement, the socalled classic appearance of a hepatic abscess, with central low attenuation and peripheral ring enhancement $(n=5)$ (Fig. 4). Thus, 96\% of the abscesses in this series showed an atypical appearance on imaging. On CT, 26\% were type $1 ; 46 \%$, type $2 \mathrm{a} ; 14 \%$, type $2 \mathrm{~b}$; and $3 \%$, type 3 . On MRI, $22 \%$ were type $1 ; 44 \%$, type $2 \mathrm{a} ; 24 \%$, type $2 \mathrm{~b}$; and $10 \%$, type 3 . Thus, $\mathrm{CT}$ and MRI findings showed a very similar distribution. Note that approximately $60 \%$ of the scans were obtained with helical CT. The CT appearance of abscesses imaged with MDCT was qualitatively similar to the that on the images obtained before the helical CT era.

A relationship was found between lesion size and the pattern of enhancement (Fig. 5). If the size of the abscess was $1 \mathrm{~cm}$ or less, most of the abscesses (40/64, or $62.5 \%$ ) displayed homogeneous and central enhancement (type 1). If the size of the abscess was $1-3 \mathrm{~cm}$, most of the abscesses $(28 / 35$, or $80 \%)$ showed incomplete central enhancement with small central hypoenhancing areas (type 2a). Thirty-seven of 40 (92.5\%) of hepatic abscesses larger than 3 $\mathrm{cm}$ appeared heterogeneous in enhancement and multiloculated with peripheral enhancement (types $2 \mathrm{a}$ and $2 \mathrm{~b}$ ). Type 3 , the most common appearance of abscesses in the general population, was distinctly unusual in this series of CGD patients. These lesions tended to be larger with measurements of $2.5,2.8,4,4$, and $5 \mathrm{~cm}$ for a mean of $3.7 \mathrm{~cm}$ (range, $2.5-5 \mathrm{~cm}$ ).

\section{$M R I$}

On T1-weighted MR images, abscesses showed homogeneous low signal intensity relative to the surrounding liver. On STIR and fat-suppressed T2-weighted images, the abscesses showed heterogeneously high signal intensity. The enhancement patterns on MRI were similar to the patterns described for CT (Fig. 6).

Because of the retrospective nature of this study, not all patients had both CT and MRI. Therefore, a comparison of sensitivities was not possible.

\section{Additional Findings Calcifications}

Approximately $33 \%$ (8/24) of patients who had had abscesses in the past showed punctate calcifications within the hepatic parenchyma at the site of the prior abscess (Fig. 7). When followed over time, the number of hepatic calcifications increased related to repeated infections with subsequent granuloma formation.

\section{Postoperative Findings}

Surgical drainage was usually required for treatment because the semisolid nature of the lesions made them unsuitable for percutaneous drainage. Several attempts were made to drain these abscesses percutaneously early in our experience but these proved unsuccessful, and since then surgery has become the standard method of treatment [12]. As a result, these patients showed extensive postoperative changes (lobulated contour and scarring) during the follow-up period.

\section{Unusual Features}

The individual severity of hepatic abscesses varied significantly among patients. One patient presented with five large hepatic abscesses requiring surgical drainage and two intrasplenic abscesses in a subcapsular region. He died related to an inferior vena cava thrombosis 3 months after surgery for the hepatosplenic abscesses. Another patient developed more than 20 small abscesses in the liver, each measuring less than $1 \mathrm{~cm}$, during a single episode. 


\section{Imaging of Hepatic Abscess in Chronic Granulomatous Disease}
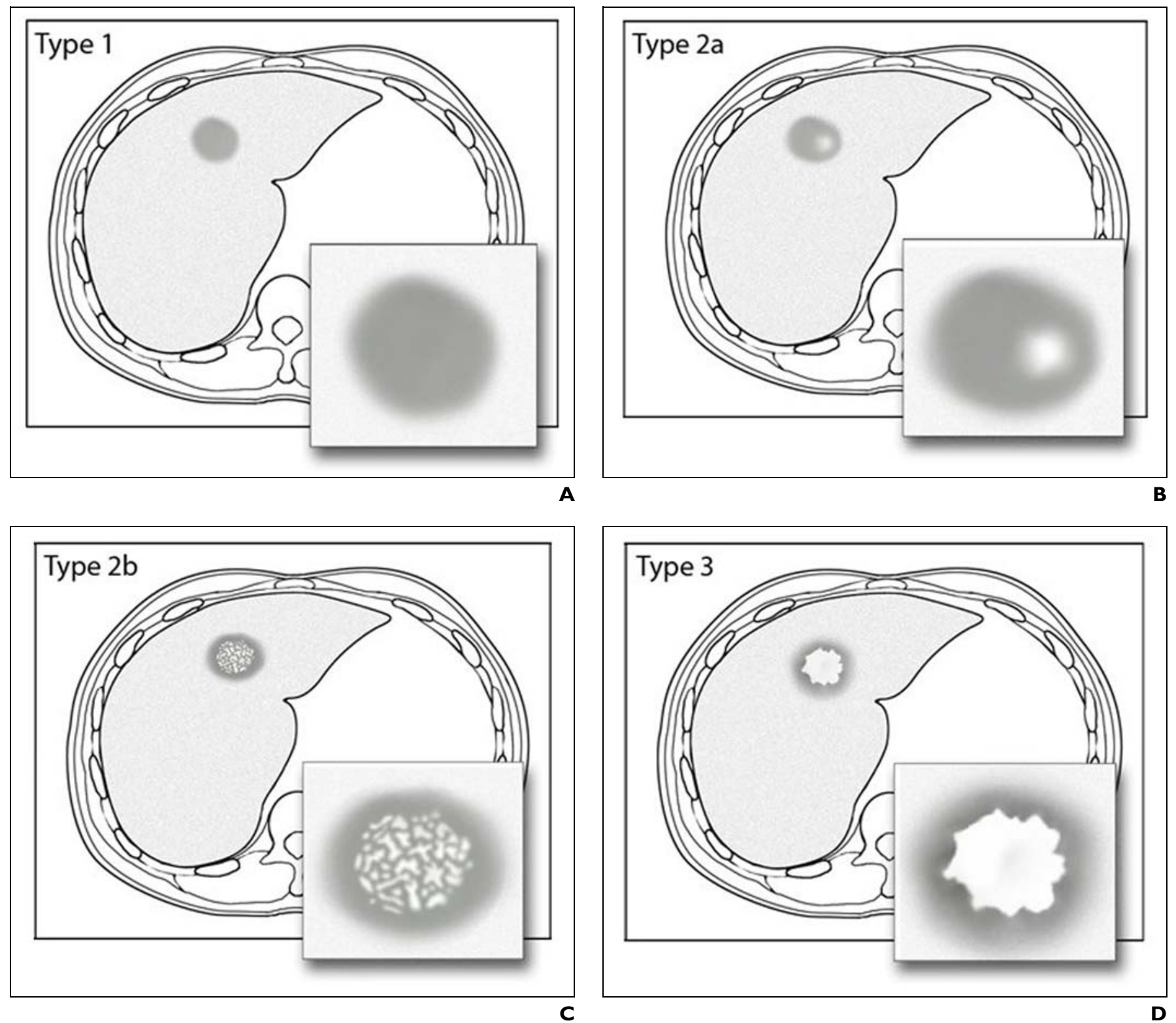

Fig. 3-Patterns of enhancement on CT and MRI of hepatic abscess in patients with chronic granulomatous disease.

A, Drawing shows type 1, complete central enhancement.

B and C, Drawings show type 2, incomplete central enhancement. Type 2 is subdivided into type $2 a(B)$, small central area with poor enhancement, and type $2 b$ (C), peripheral enhancement and multiseptal core enhancement suggesting multiloculated abscess.

D, Drawing shows type 3 , peripheral ring enhancement surrounding necrotic liquefied cavity.

One patient with a hepatic abscess also presented with a left paraaortic hypodense mass with multiple enlarged retroperitoneal lymph nodes in the gastrohepatic ligament consistent with a clinical diagnosis of retroperitoneal adenitis. Among the 24 patients with hepatic abscess, 8 also developed splenic abscesses, and 2 cases of retroperitoneal adenitis were encountered.

\section{Discussion}

Pyogenic hepatic abscesses are usually caused by gram-negative bacilli [3, 4]. An inoculum of bacteria leads to an initial inflammatory response; as macrophages destroy healthy and infected tissue, the center of the nascent abscess necroses and its contents liquefy. The abscess becomes walled off from healthy tissue and a low-grade inflammatory response develops. Sporadic hepatic abscesses typically occur as single events because of trauma or sepsis, and recurrences are unusual. Because of their liquefied centers, they are often amenable to percutaneous drainage.

CGD, however, is a rare condition in which neutrophils and macrophages are unable to generate superoxide $[6,8]$. As a result, the lungs and liver develop recurrent ab- 


\section{Garcia-Eulate et al.}
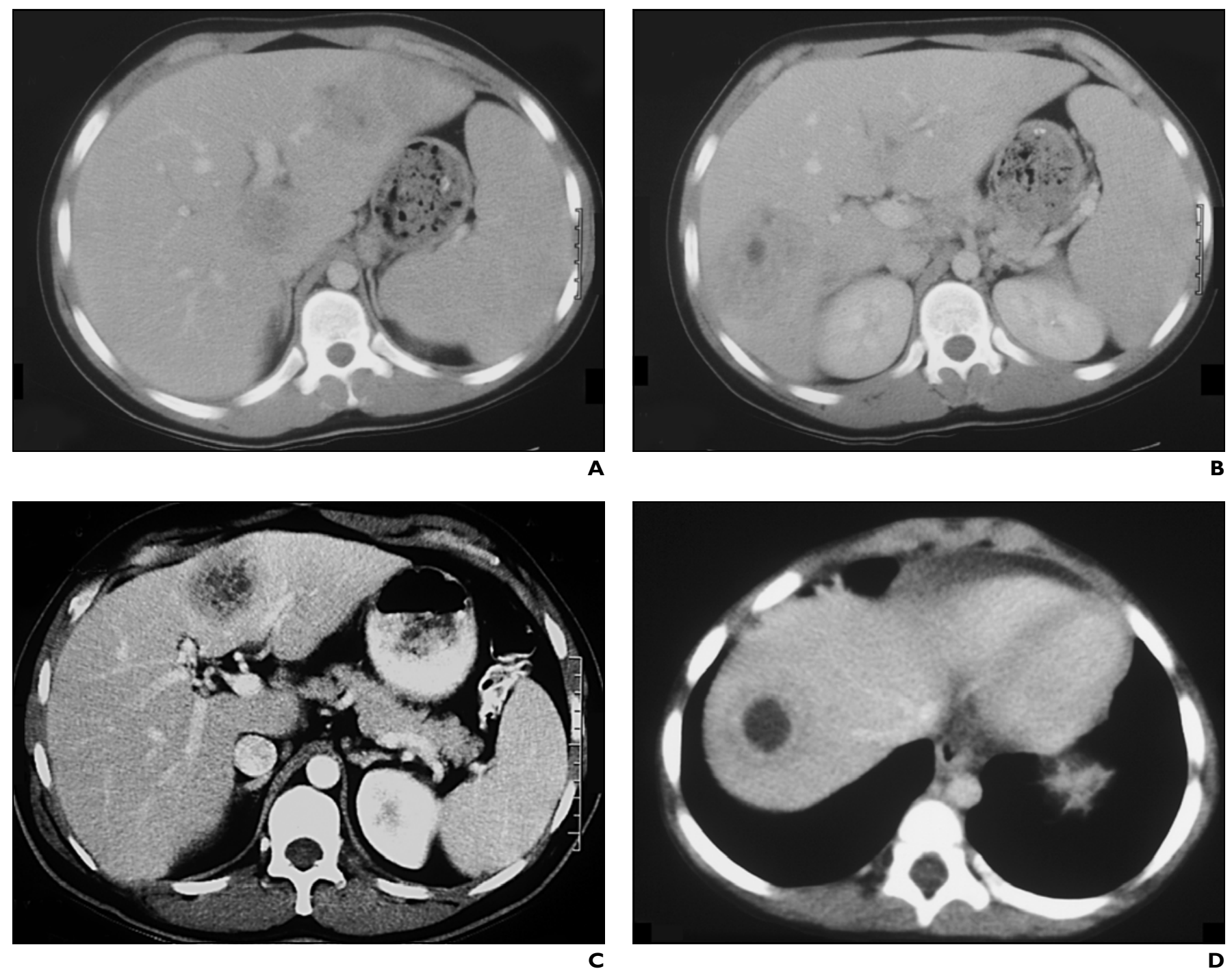

Fig. 4-Hepatic abscess in patients with chronic granulomatous disease showing different patterns of enhancement.

A, 15-year-old boy with homogeneous enhancement (type 1).

B, Same patient and episode as A (type 2a): predominance of homogeneous enhancement with small central area with poor enhancement.

C, 25-year-old man with peripheral and multiseptal enhancement (type 2b).

D, 3-year-old boy with central low attenuation and peripheral ring enhancement (type 3).

scesses and infections. As we have shown, CGD abscesses are often multiple during each episode, and patients with CGD often have multiple episodes of hepatic infection leading to progressive calcification and scarring. Unlike the sporadic abscess, the liver abscess in CGD is typically caused by Staphylococcus aureus and some fungi. These infections result in the formation of granulomas, some of which scar and calcify. Others lead to clinical symptoms of abscess and develop a granulomatous reaction that is surrounded by granulation tissue.
The hepatic abscesses associated with CGD have several imaging characteristics that differentiate them from most sporadic hepatic abscesses. Whereas sporadic abscesses usually show central cystic cavities $[4,13]$, the abscesses associated with CGD tend to remain homogeneously enhancing while small and then develop multiple locules separated by thick enhancing septations as they enlarge [12]. There is often an intense halo of enhancement around the abscess, likely representing granulation tissue. The locules may be quite small, and the pre- dominant appearance may be one of a solid vascular lesion.

As a consequence of this reactive tissue architecture, it is difficult to drain CGD hepatic abscesses percutaneously, and surgical intervention with manual débridement may be required [11]. Because patients with CGD often have poor healing confounded by granuloma formation, repeated surgeries may lead to severe scar formation and deformation of the hepatic contour.

CGD patients who develop fever without a clear source should undergo CT of the chest 


\section{Imaging of Hepatic Abscess in Chronic Granulomatous Disease}

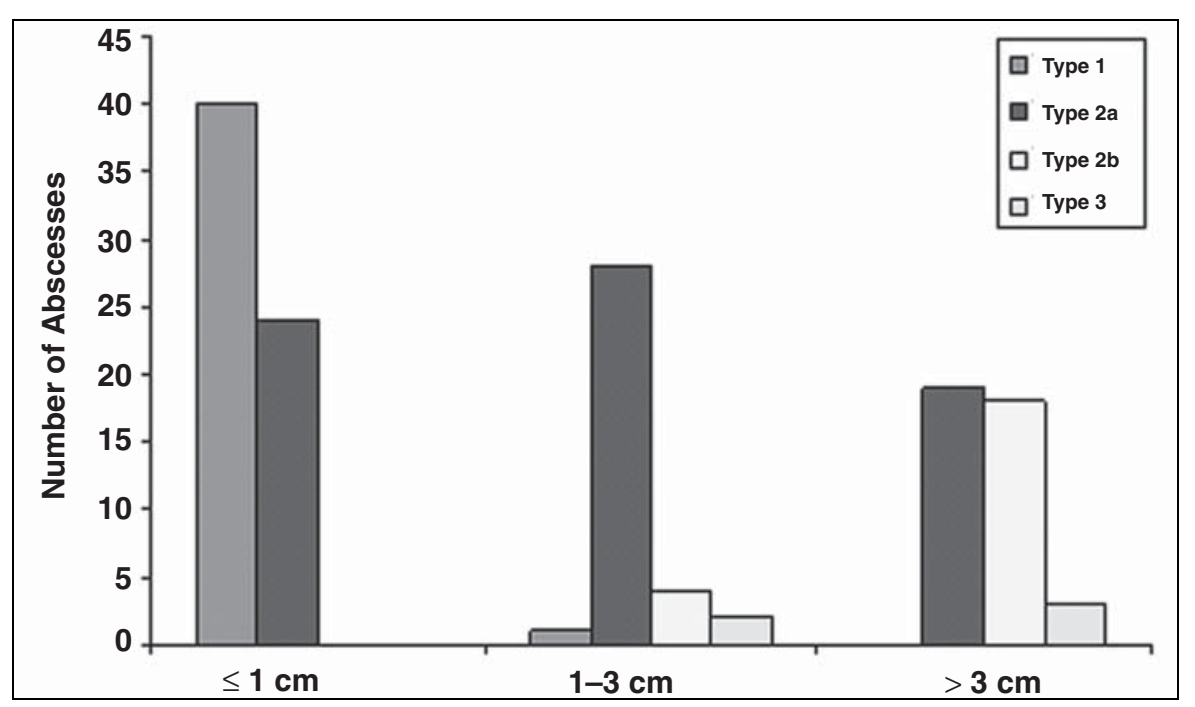

Fig. 5-This graph depicts relative frequency of each type of abscess based on lesion size. Most small abscesses $(\leq 1 \mathrm{~cm})$ display homogeneous enhancement (type 1), whereas $80 \%$ of abscesses between more than $1 \mathrm{~cm}$ and $3 \mathrm{~cm}$ show incomplete central enhancement with small central hypoenhancing area (type 2a). Most abscesses larger than $3 \mathrm{~cm}$ show either type 2a (incomplete central enhancement) or type $2 b$ (multiloculated with peripheral enhancement).
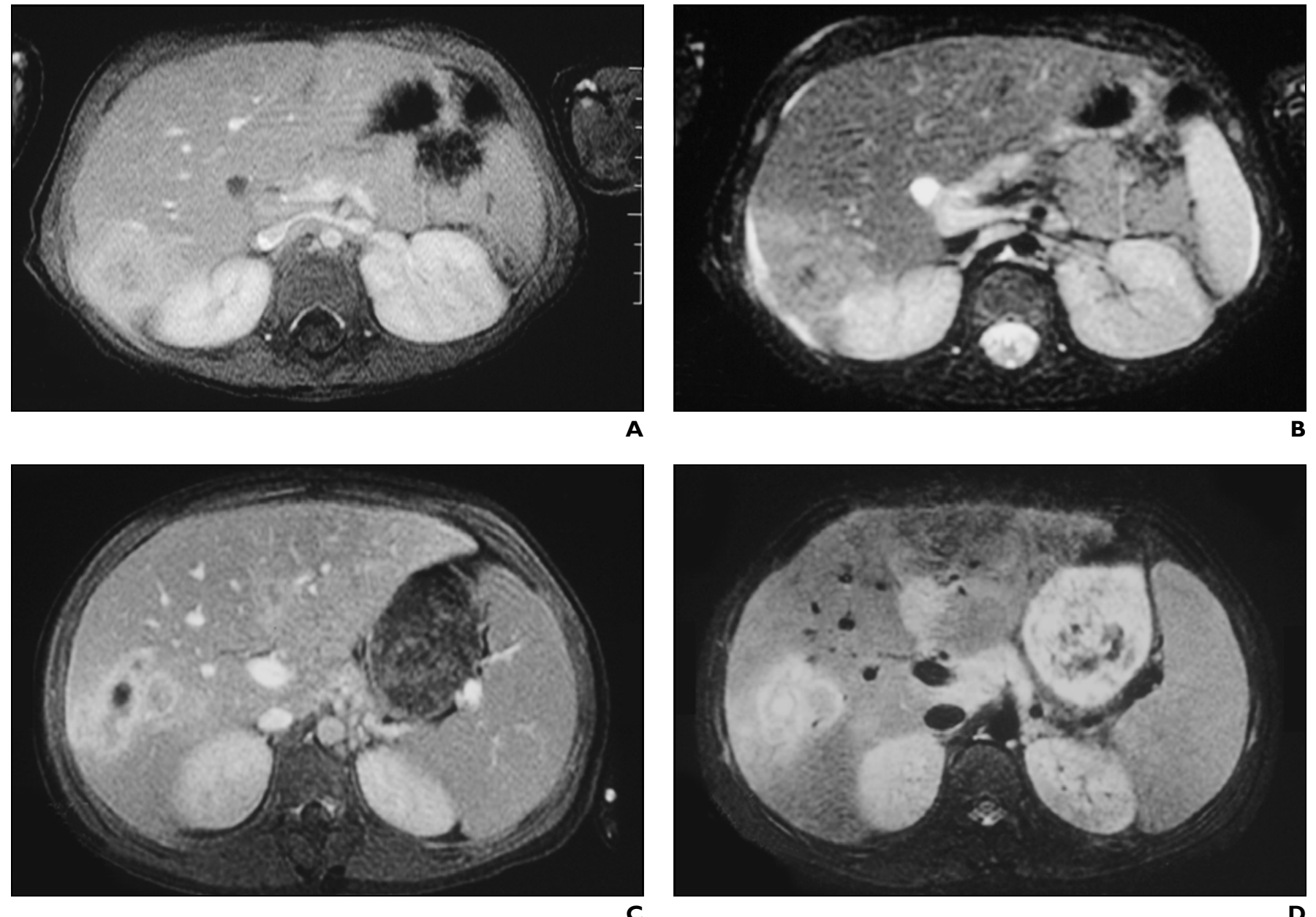

Fig. 6-Patterns of enhancement of hepatic abscess in patients with chronic granulomatous disease (CGD) on MRI are similar to CT.

$\mathbf{A}$ and B, 3-year-old boy with homogeneous enhancement hepatic abscess (type 1) on T1-weighted contrast-enhanced (A) and mild increased signal intensity on STIR (B) images. C and D, 15-year-old boy presents with enhancing hepatic lesion and low-signal central area (type 2a) on T1-weighted contrast-enhanced (C) and moderately increased signal intensity on STIR (D) images.

(Fig. 6 continues on next page) 


\section{Garcia-Eulate et al.}
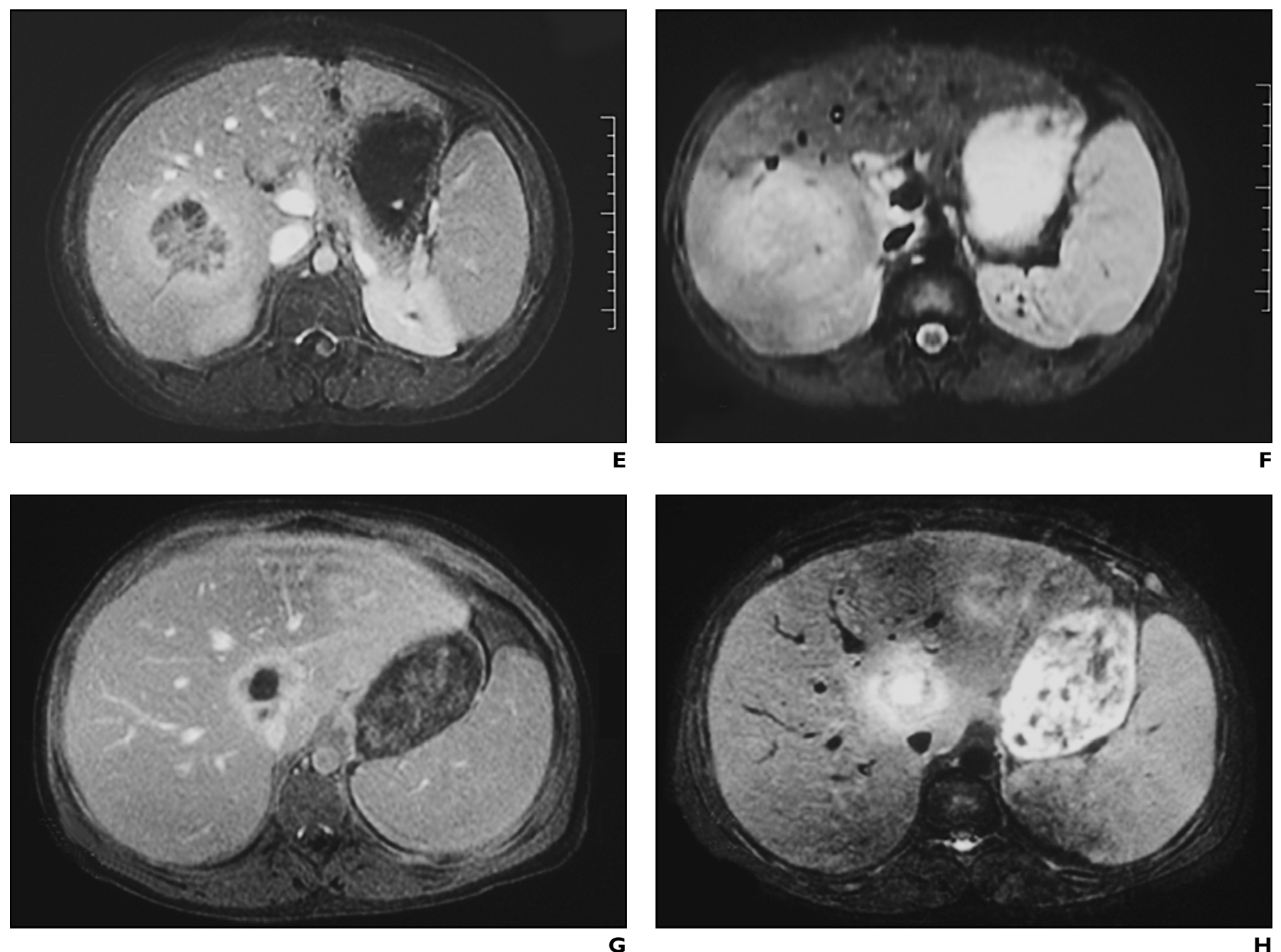

Fig. 6 (continued)—Patterns of enhancement of hepatic abscess in patients with chronic granulomatous disease (CGD) on MRI are similar to CT.

$\mathbf{E}$ and $\mathbf{F}$, 11-year-old boy shows hepatic abscess with peripheral and septal enhancement (type 2b) on T1-weighted contrast-enhanced image (E). On STIR image (F), this lesion shows moderate but homogeneous intensity.

$\mathbf{G}$ and $\mathbf{H}, 15$-year-old boy presents so-called classic appearance of abscess with enhancing ring and necrotic central area (type 3) on T1-weighted contrast-enhanced image (G). On STIR image (H), signal intensity is slightly higher centrally within cavity compared with periphery.

and abdomen. The latter should be performed after IV contrast medium to help identify typical lesions. A single venous phase scan is usually sufficient in such patients because the septal thickening requires approximately 1 minute to enhance. MRI of the abdomen should include a T1-weighted, T2-weighted, and gadolinium-enhanced T1-weighted axial scan and should be performed if CT is negative or equivocal. MRI should also be used in patients with a history of iodinated contrast allergy or renal insufficiency. MRI does not expose affected children to radiation. CGD patients often undergo multiple radiographic examinations, and cumulative ionizing radiation may be a cause for concern. Therefore, MRI is a radiation-free alternative to CT for diagnosing and following hepatic abscesses. Sonography, which in theory is an ideal alternative, is limited because the lesions are often isoechoic with the liver parenchyma. In addition, because the liver is usually scarred, the sonographic appearance is complex. The abscesses are not cystic, so they can be difficult to distinguish from scars and calcifications, which are common. Moreover, in many children, prior scarring and surgical defects make differentiation of active and inactive abscesses problematic without the use of contrast medium. We have no experience with sonographic contrast agents in this setting, but they are a reasonable consideration. In reality, when patients present with fever and a history of CGD, the first test obtained is CT of the chest and abdomen to exclude both pulmonary and abdominal infection. If the result of the CT is clearly negative, no further testing is needed. If, however, some doubt exists, the findings can be confirmed with hepatic MRI.

We propose a classification system for hepatic abscesses in CGD based on what appears to be the evolution of such lesions. The major purpose of this classification is to educate imagers regarding the different patterns of CGD-associated abscesses. In this classification, type 1 lesions are homogeneously enhancing, type 2 lesions show incomplete or septated central radiolucency with a thick enhancing wall, and type 3 lesions contain a liq- 


\section{Imaging of Hepatic Abscess in Chronic Granulomatous Disease}
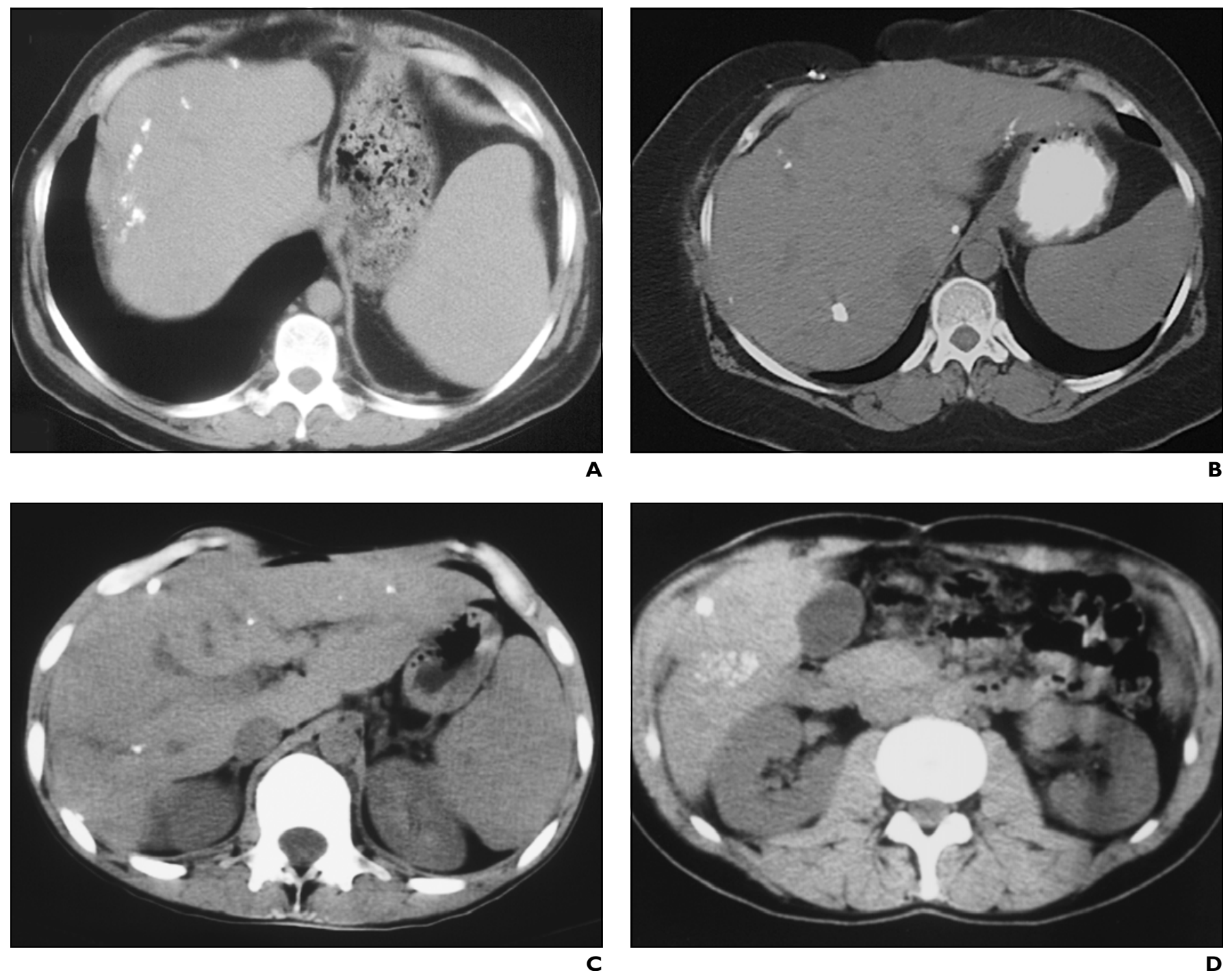

Fig. 7-Long-term sequelae of hepatic abscesses in chronic granulomatous disease (CGD). Calcifications scattered within hepatic parenchyma of different patients with CGD.

A, 37-year-old man with punctate calcifications near dome of liver because of previous abscess associated with scarring.

B, 39-year-old man with scattered punctate calcifications without scarring.

C, 12-year-old boy also with scattered punctate calcifications, indicating this process can occur early in life.

D, 39-year-old man showing masslike calcifications within liver at site of previous abscess.

uefied center with a thin enhancing wall. Small lesions ( $1 \mathrm{~cm}$ or less) are usually homogeneously enhancing (type 1). Intermediate lesions ( $>1-3 \mathrm{~cm}$ in diameter) often have nonenhancing small central areas (type 2a). Larger lesions $(>3 \mathrm{~cm}$ ) show either type $2 \mathrm{a}$ (incomplete central enhancement) or type $2 b$ (multiloculated with peripheral enhancement) characteristics. The most unusual appearance for a CGD hepatic abscess is the most common appearance for a sporadic he- patic abscess: a cavitary lesion with an enhancing rim (type 3) seen in only approximately $3 \%$ of the abscesses in this series.

This study has a number of limitations. Because the imaging was performed over 20 years, imaging technology was not consistent. Partial volume averaging effects on older scanners with 1-cm collimation could have resulted in a higher percentage of lesions considered to be type 1 versus type 2 . However, the fundamental types of ab- scesses described here will not vary significantly. A substantial proportion of the patients in this series (39-70\%) were scanned with modern equipment. In addition, we did not routinely obtain multiphasic CT or MRI after contrast administration. This is because the patients are usually children, and attempts are made to minimize radiation exposure or time in the scanner. It might be interesting to examine dynamic enhanced MRI of such lesions, however. 


\section{Garcia-Eulate et al.}

In conclusion, hepatic abscesses in patients with CGD have characteristic imaging appearances that include homogeneously enhancing lesions, a vascular abscess wall, and multiloculated chambers. Small solid enhancing hepatic lesions in a young patient with CGD are highly indicative of acute hepatic abscesses that require early treatment. These lesions should not be mistaken for other noninfectious vascular hepatic lesions such as adenomas or metastatic disease. Moreover, because they are nonliquefied, they are difficult to drain percutaneously. The diagnosis can be made on CT or MRI, but the latter shows smaller lesions more readily and spares radiation exposure in young children. Repeated abscess formation leads to hepatic scarring and calcifications, which may compromise hepatic function. Although there is overlap with sporadic hepatic abscess, in the appropriate setting the findings described here are highly suggestive of CGD-related hepatic abscess.

\section{References}

1. Mortele KJ, Segatto E, Ros PR. The infected liver: radiologic-pathologic correlation. RadioGraphics 2004; 24:937-955

2. Balci NC, Semelka RC, Noone TC, et al. Pyogenic hepatic abscesses: MRI findings on T1- and T2-weighted and serial gadolinium-enhanced gradient-echo images. J Magn Reson Imaging 1999; 9:285-290

3. Kawamoto S, Soyer PA, Fishman EK, Bluemke DA. Nonneoplastic liver disease: evaluation with CT and MR imaging. RadioGraphics 1998; 18:827-848

4. Mortele KJ, Ros PR. Cystic focal liver lesions in the adult: differential CT and MR imaging features. $R a$ dioGraphics 2001; 21:895-910

5. Barreda R, Ros PR. Diagnostic imaging of liver abscess. Crit Rev Diagn Imaging 1992; 33:29-58

6. Heyworth PG, Cross AR, Curnutte JT. Chronic granulomatous disease. Curr Opin Immunol 2003; 15:578-584

7. Curnutte JT. Chronic granulomatous disease: the solving of a clinical riddle at the molecular level. Clin Immunol Immunopathol 1993; 67:S2-S15

8. Segal BH, Leto TL, Gallin JI, Malech HL, Holland SM. Genetic, biochemical, and clinical features of chronic granulomatous disease. Medicine (Baltimore) 2000; 79:170-200

9. Rosenzweig SD, Holland SM. Phagocyte immunodeficiencies and their infections. JAllergy Clin Immunol 2004; 113:620-626

10. Eckert JW, Abramson SL, Starke J, Brandt ML. The surgical implications of chronic granulomatous disease. Am J Surg 1995; 169:320-323

11. Chen LE, Minkes RK, Shackelford PG, Strasberg SM, Kuo EY, Langer JC. Cut it out: managing hepatic abscesses in patients with chronic granulomatous disease. J Pediatr Surg 2003; 38:709-713

12. Lublin M, Bartlett DL, Danforth DN, et al. Hepatic abscess in patients with chronic granulomatous disease. Ann Surg 2002; 235:383-391

13. Rustgi AK, Richter JM. Pyogenic and amebic liver abscess. Med Clin North Am 1989; 73:847-858

\section{FOR YOUR INFORMATION}

This article is available for CME credit. See CME data for this article at www.ajronline.org or visit www.arrs.org for more information. 